\title{
A numerical study on time fractional Fisher equation using an extended cubic B-spline approximation
}

\author{
Tayyaba Akram ${ }^{\mathrm{a}, *}$, Muhammad Abbas $^{\mathrm{b}}$, Ajmal Ali $^{\mathrm{a}}$ \\ a School of Mathematical Sciences, Universiti Sains Malaysia, 11800 Penang, Malaysia. \\ ${ }^{b}$ Department of Mathematics, University of Sargodha, 40100 Sargodha, Pakistan.
}

\begin{abstract}
A new extended cubic B-spline approximation for the numerical solution of the time-fractional fisher equation is formed and examined. The given non-linear partial differential equation through substitution is converted into a linear partial differential equation through substitution, using Taylor's series expansion. The time-fractional derivative is approximated in Caputo's sense while the space dimension is calculated using a new extended cubic B-spline. The proposed numerical technique is shown to be unconditionally stable and convergent. The errors are used for measuring the accuracy of the proposed technique. The graphical and numerical results are presented to illustrate the performance of the technique.
\end{abstract}

Keywords: Time fractional Fisher equation, extended cubic B-spline, Caputo's derivative, stability, convergence.

2020 MSC: 35R10, 35R12, 65M06, 65M12.

(C)2021 All rights reserved.

\section{Introduction}

In recent decades, fractional calculus has created its eminent attention in multiple fields of viscoelasticity [14], bioengineering [28], control theory, aerodynamics, thermodynamics, electro-magnetics, signal processing, chemistry, and finance [22, 23]. Different numerical methods have been implemented and observed for differential equations containing fractional order derivative in the form of the RiemannLiouville, Caputo sense, Atangana-Baleanu [2, 6, 15, 26, 29]. Delay differential equations can be taken in account as the generalization of the common differential equations, that are being used for modelling the physical systems with memory. The delay is a vital element of the physical systems such as kinetics, controllers, signal processing, and damping conduct of viscoelastic materials. Time fractional Fishers equation (TFFE) also explains the space and time propagation of a virile gene in an infinite medium [18]. Consider a TFFE with small delay [18, 33]:

$$
{ }_{0}^{C} D_{t}^{\beta} v(u, t)=D_{u u} v(u, t)+6 v(u, t)(1-v(u, t)), \quad u \in[a, b], \quad t \in[0, T], \quad 0<\beta<1,
$$

\footnotetext{
*Corresponding author

Email address: tayyaba.akram2020@gmail.com (Tayyaba Akram)
}

doi: $10.22436 /$ jmcs.022.01.08

Received: 2020-03-30 Revised: 2020-04-29 Accepted: 2020-05-16 
subject to Initial and boundary conditions

$$
v(u, 0)=A_{1}(u), v(a, t)=B_{1}(t), v(b, t)=B_{2}(t) .
$$

The mathematical model of TFFE, used for multiple dimensions of physical peculiarities, is a partial differential equation developed by substituting the time derivative with a fractional derivative of order within the classical Fisher Equation (FE) [20]. Equation (1.1) with (1.2) is recognized as FE, explains the dissemination of a virile mutant in an enormously lengthy habitation. It also characterizes the model equation for the development of a neutron population within a nuclear reactor and serves as a prototype model for a proliferating flame. The non-linear model problem has attained much importance due to its usage in numerous biological and chemical processes.

Several researchers have considered FE. A pseudo-spectral method has been developed to solve FE numerically by Olmos and Shizgal [27]. Mickens [24] and Uddin [31] have proposed finite difference techniques to obtain the numerical solutions of FE. Many methods such as residual power series method [18], Laplace Adomian decomposition method [1], reduced differential transform method [1], and homotopy perturbation method [33] have been employed to obtain the solutions for TFFE. A new iterative method based on residual power series for solving TFFE has been presented by Qurashi et al. [12]. Khader and Saad [21] proposed a numerical method to solve fractional FE by Chebyshev collocation method. Veeresha et al. [32] have obtained a numerical technique for solving TFFE via q-homotopy analysis transform method. Akgül et al. [5] derived solutions of fractional gas dynamics equation by a new technique using reproducing kernel method. Akgül [3] investigated boundary layer flow of a PowellEyring non-Newtonian fluid by reproducing kernel Hilbert space method. Akgül [4] proposed solutions of the linear and nonlinear differential equations within the generalized fractional derivatives.

Several researchers have used B-spline collocation method to solve linear and non-linear fractional partial differential equations (FPDEs). There are a large number of numerical techniques already established for solving FPDEs. However, as far as we are aware there is no such study on the use of B-splines for solving TFFE. The quadratic B-spline Galerkin method and cubic B-spline (CBS) finite element method have been utilized to solve FPDEs numerically by Esen and Tasbozan [19, 30]. Arshed [13] presented a numerical method based on quintic B-spline approach to solve hyperbolic FPDEs. Mohyud-din [25] obtained the solution of fractional advection diffusion equation by ECuBS approach. Akram et al. [8,9] presented numerical solutions of linear FPDEs via ECuBS method and Caputo fractional derivative (CFD). Akram et al. $[7,11]$ derived ECuBS method for the solution of time fractional Burgers and time fractional Klein-Gordon equations. In this research paper, we will identify numerical solution for the TFFE using new ECuBS approach in Caputo sense.

This research paper is divided and explained in various sections. The preliminaries containing the definition and basic function are presented in Section 2. Section 3 demonstrates the description of ECuBS method. Von Neumann stability analysis is comprised in Section 4 while the convergence is derived in Section 5. Numerical and graphical results are shown in Section 6. Lastly, concluding remarks are mentioned in Section 7.

\section{Preliminaries}

Definition 2.1. The CFD of order $\beta$ of a $g(t)$ is defined as [17]:

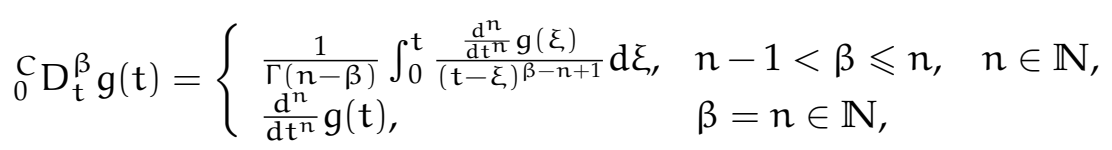

where ${ }_{0}^{C} D_{t}^{\beta}$ denotes the CFD.

\subsection{Basis function}

Let $\left\{u_{i}\right\}$ be a uniform partitioning of a finite interval $[a, b]$ with $i \in \mathbb{Z}$. Therefore, assumed interval is divided into $N$ equal subintervals at the knots as $u_{i}=u_{0}+i h$, where $h$ is a step size. The ECuBS 
functions at the $u_{i}$ over the assumed interval is described as follows;

$$
E_{i}(u, \eta)=\frac{1}{24 h^{4}} \begin{cases}4 h(1-\eta)\left(u-u_{i-2}\right)^{3}+3 \eta\left(u-u_{i-2}\right)^{4}, & u \in\left[u_{i-2}, u_{i-1}\right), \\ (4-\eta) h^{4}+12 h^{3}\left(u-u_{i-1}\right)+6 h^{2}(2+\eta)\left(u-u_{i-1}\right)^{2} & \\ -12 h\left(u-u_{i-1}\right)^{3}-3 \eta\left(u-u_{i-1}\right)^{4}, & u \in\left[u_{i-1}, u_{i}\right), \\ (4-\eta) h^{4}+12 h^{3}\left(u_{i+1}-u\right)+6 h^{2}(2+\eta)\left(u_{i+1}-u\right)^{2} & \\ -12 h\left(u_{i+1}-u\right)^{3}-3 \eta\left(u_{i+1}-u\right)^{4}, & u \in\left[u_{i}, u_{i+1}\right), \\ 4 h(1-\eta)\left(u_{i+2}-u\right)^{3}+3 \eta\left(u_{i+2}-u\right)^{4}, & u \in\left[u_{i+1}, u_{i+2}\right), \\ 0, & \text { otherwise, }\end{cases}
$$

where $i=-1(1) N+1, \eta \in \mathbb{R}$ is a free parameter within the interval $[-8,1]$ and $u \in \mathbb{R}$ is a variable. For $\eta \in[-8,1]$, the ECuBS and CBS basis preserve same properties. The ECuBS becomes CBS for $\eta=0$. For a smooth function $v(u, t)$ there is a unique ECuBS $V(u, t)$, that fulfills the prescibed interpolating conditions, such that

$$
V(u, t)=\sum_{i=j-1}^{j+1} \delta_{i}^{k}(t) E B_{i}(u, \eta),
$$

where $\delta_{j}(t)$ 's are time dependent unknown coefficients and are to be calculated by some particular restrictions. The ECuBS functions (2.1) and equation (2.2) produce the following relations

$$
\begin{aligned}
& V_{j}(u, t)=\sum_{i=j-1}^{j+1} \delta_{i}(t) E B_{i}(u, \eta)=\left(\frac{4-\eta}{24}\right) \delta_{j-1}+\left(\frac{8+\eta}{12}\right) \delta_{j}+\left(\frac{4-\eta}{24}\right) \delta_{j+1}, \\
& V_{j}^{\prime}(u, t)=\sum_{i=j-1}^{j+1} \delta_{i}(t) E B_{i}^{\prime}(u, \eta)=\left(-\frac{1}{2 h}\right) \delta_{j-1}+\left(\frac{1}{2 h}\right) \delta_{j+1} .
\end{aligned}
$$

The following new approximation has formulated in [10]

$$
V_{j}^{\prime \prime}(u, t)=\frac{1}{24 h^{2}}\left\{\begin{array}{cl}
2(14-\eta) \delta_{-1}+3(3 \eta-22) \delta_{0}+8(7-2 \eta) \delta_{1} \\
+14(\eta-2) \delta_{2}+6(2-\eta) \delta_{3}+(\eta-2) \delta_{4}, \\
(2-\eta) \delta_{j-2}+4(4+\eta) \delta_{j-1}-6(6+\eta) \delta_{j} \\
+4(4+\eta) \delta_{j+1}+(2-\eta) \delta_{j+2}, & \text { for } i=0, \\
(\eta-2) \delta_{N-4}+6(2-\eta) \delta_{N-3}+14(\eta-2) \delta_{N-2} & \text { for } i=1(1) N-1, \\
+8(7-2 \eta) \delta_{N-1}+3(3 \eta-22) \delta_{N}+2(14-\eta) \delta_{N+1}, & \text { for } i=N .
\end{array}\right.
$$

\section{Derivation of the method}

In this segment, we propose the numerical technique to solve the TFFE in Caputo's sense. For time discretization, take uniform partitioning of $[0, T]$ as $t_{k}=t_{0}+k \tau$ with $\tau=t_{k+1}-t_{k}$ for $k=0,1, \ldots, M-1$. The discretized form of CFD is described as follows

$$
\frac{\partial^{\eta} v\left(u, t_{k+1}\right)}{\partial t^{\eta}}=\frac{1}{\Gamma(2-\eta)} \sum_{p=0}^{k} b_{p} \frac{v\left(u, t_{k+1-p}\right)-v\left(u, t_{k-p}\right)}{\tau^{\eta}}+e_{\tau}^{k+1},
$$

where $b_{p}=(p+1)^{1-\eta}-p^{1-\eta}$. The coefficients $b_{p}$ satisfy the following conditions

$$
\left\{\begin{array}{l}
b_{0}=1, \\
b_{0}>b_{1}>b_{2}>\cdots>b_{p}, b_{p} \rightarrow 0 \text { as } p \rightarrow \infty, \\
b_{p}>0 \text { for } p=0,1, \ldots, k, \\
\sum_{p=0}^{k}\left(b_{p}-b_{p+1}\right)+b_{k+1}=\left(1-b_{1}\right)+\sum_{p=1}^{k-1}\left(b_{p}-b_{p+1}\right)+b_{k}=1 .
\end{array}\right.
$$


The truncation error $e_{\tau}^{k+1}$ is bounded, i.e.,

$$
\left|e_{\tau}^{k+1}\right| \leqslant C \tau^{2-\beta}
$$

where $C$ is a constant. Apply $\theta$ weighted method and equation (3.1) in equation (1.1), we obtain

$$
\frac{\tau^{-\beta}}{\Gamma(2-\beta)} \sum_{p=0}^{k} b_{p}\left[v_{j}^{k+1-p}-v_{j}^{k-p}\right]=\theta\left(v_{\mathfrak{u u}}^{k+1}+6 v^{k+1}-6\left(v^{2}\right)^{k+1}\right)+(1-\theta)\left(v_{\mathfrak{u u}}^{k}+6 v^{k}-6\left(v^{2}\right)^{k}\right) .
$$

Linearize the non-linear term as [10]

$$
\left(v^{2}\right)^{k+1}=2 v^{k} v^{k+1}-v^{k} v^{k}+\mathrm{O}(\tau)^{2} .
$$

Substituting (3.3) in (3.2) and $\theta=1$, we get

$$
\frac{\tau^{-\beta}}{\Gamma(2-\beta)} \sum_{p=0}^{k} b_{p}\left[v_{j}^{k+1-p}-v_{j}^{k-p}\right]=v_{u u}^{k+1}+6 v^{k+1}-12 v^{k} v^{k+1}
$$

After some simplifications and substituting the relations (2.3)-(2.5) in the above equation, we obtain

$$
V_{j}^{k+1}-6 r V_{j}^{k+1}-r\left(V^{\prime \prime}\right)_{j}^{k+1}+12 r V_{j}^{k} V_{j}^{k+1}=V_{j}^{k}+6 r\left(V_{j}^{k}\right)^{2}-\sum_{p=1}^{k} b_{p}\left[V_{j}^{k-p+1}-V_{j}^{k-p}\right],
$$

where $r=\tau^{\beta} \Gamma(2-\beta)$. The above equation (3.4) can be written as

$$
V_{j}^{k+1}-6 r V_{j}^{k+1}-r\left(V^{\prime \prime}\right)_{j}^{k+1}+12 r V_{j}^{k} V_{j}^{k+1}=b_{k} V_{j}^{0}+6 r\left(V_{j}^{k}\right)^{2}+\sum_{p=0}^{k-1}\left(b_{p}-b_{p+1}\right) V_{j}^{k-p} .
$$

By expanding the above relation for $j=0,1, \ldots, N$, we obtain the following linear equations having $N+3$ unknowns.

$$
\begin{gathered}
(1-6 r)\left[\left(\frac{4-\eta}{24}\right) \delta_{-1}^{k+1}+\left(\frac{8+\eta}{12}\right) \delta_{0}^{k+1}+\left(\frac{4-\eta}{24}\right) \delta_{1}^{k+1}\right]+12 r V_{0}^{k}\left[\left(\frac{4-\eta}{24}\right) \delta_{-1}^{k+1}+\left(\frac{8+\eta}{12}\right) \delta_{0}^{k+1}\right. \\
\left.+\left(\frac{4-\eta}{24}\right) \delta_{1}^{k+1}\right]-\frac{r}{24 h^{2}}\left[2(14-\eta) \delta_{-1}^{k+1}+3(3 \eta-22) \delta_{0}^{k+1}+8(7-2 \eta) \delta_{1}^{k+1}\right. \\
\left.+14(\eta-2) \delta_{2}^{k+1}+6(2-\eta) \delta_{3}^{k+1}+(\eta-2) \delta_{4}^{k+1}\right] \\
=b_{k}\left[\left(\frac{4-\eta}{24}\right) \delta_{-1}^{0}+\left(\frac{8+\eta}{12}\right) \delta_{0}^{0}+\left(\frac{4-\eta}{24}\right) \delta_{1}^{0}\right]+6 r V_{0}^{k}\left[\left(\frac{4-\eta}{24}\right) \delta_{-1}^{k}+\left(\frac{8+\eta}{12}\right) \delta_{0}^{k}\right. \\
\left.+\left(\frac{4-\eta}{24}\right) \delta_{1}^{k}\right]+\sum_{p=0}^{k-1}\left(b_{p}-b_{p+1}\right)\left[\left(\frac{4-\eta}{24}\right) \delta_{-1}^{k-p}+\left(\frac{8+\eta}{12}\right) \delta_{0}^{k-p}+\left(\frac{4-\eta}{24}\right) \delta_{1}^{k-p}\right], \\
(1-6 r)\left[\left(\frac{4-\eta}{24}\right) \delta_{j-1}^{k+1}+\left(\frac{8+\eta}{12}\right) \delta_{j}^{k+1}+\left(\frac{4-\eta}{24}\right) \delta_{j+1}^{k+1}\right]+12 r V_{j}^{k}\left[\left(\frac{4-\eta}{24}\right) \delta_{j-1}^{k+1}+\left(\frac{8+\eta}{12}\right) \delta_{j}^{k+1}\right. \\
\left.+\left(\frac{4-\eta}{24}\right) \delta_{j+1}^{k+1}\right]-\frac{r}{24 h^{2}}\left[(2-\eta) \delta_{j-2}^{k+1}+4(4+\eta) \delta_{j-1}^{k+1}-6(6+\eta) \delta_{j}^{k+1}+4(4+\eta) \delta_{j+1}^{k+1}+(2-\eta) \delta_{j+2}^{k+1}\right] \\
=b_{k}\left[\left(\frac{4-\eta}{24}\right) \delta_{j-1}^{0}+\left(\frac{8+\eta}{12}\right) \delta_{j}^{0}+\left(\frac{4-\eta}{24}\right) \delta_{j+1}^{0}\right]+6 r V_{j}^{k}\left[\left(\frac{4-\eta}{24}\right) \delta_{j-1}^{k}+\left(\frac{8+\eta}{12}\right) \delta_{j}^{k}+\left(\frac{4-\eta}{24}\right) \delta_{j+1}^{k}\right] \\
+\sum_{p=0}^{k-1}\left(b_{p}-b_{p+1}\right)\left[\left(\frac{4-\eta}{24}\right) \delta_{j-1}^{k-p}+\left(\frac{8+\eta}{12}\right) \delta_{j}^{k-p}+\left(\frac{4-\eta}{24}\right) \delta_{j+1}^{k-p}\right], \quad j=1,2, \ldots, N-1,
\end{gathered}
$$




$$
\begin{aligned}
(1-6 r) & {\left[\left(\frac{4-\eta}{24}\right) \delta_{N-1}^{k+1}+\left(\frac{8+\eta}{12}\right) \delta_{N}^{k+1}+\left(\frac{4-\eta}{24}\right) \delta_{N+1}^{k+1}\right]+12 r V_{N}^{k}\left[\left(\frac{4-\eta}{24}\right) \delta_{N-4}^{k+1}+\left(\frac{8+\eta}{12}\right) \delta_{N-3}^{k+1}\right.} \\
& \left.+\left(\frac{4-\eta}{24}\right) \delta_{N+1}^{k+1}\right]-\frac{r}{24 h^{2}}\left[2(14-\eta) \delta_{N-1}^{k+1}+3(3 \eta-22) \delta_{N}^{k+1}+8(7-2 \eta) \delta_{N-2}^{k+1}\right. \\
& \left.+14(\eta-2) \delta_{N-1}^{k+1}+6(2-\eta) \delta_{N}^{k+1}+(\eta-2) \delta_{N+1}^{k+1}\right] \\
= & b_{k}\left[\left(\frac{4-\eta}{24}\right) \delta_{N-1}^{0}+\left(\frac{8+\eta}{12}\right) \delta_{N}^{0}+\left(\frac{4-\eta}{24}\right) \delta_{N+1}^{0}\right]+6 r V_{N}^{k}\left[\left(\frac{4-\eta}{24}\right) \delta_{N-1}^{k}+\left(\frac{8+\eta}{12}\right) \delta_{N}^{k}\right. \\
& \left.+\left(\frac{4-\eta}{24}\right) \delta_{N+1}^{k}\right]+\sum_{p=0}^{k-1}\left(b_{p}-b_{p+1}\right)\left[\left(\frac{4-\eta}{24}\right) \delta_{N-1}^{k-p}+\left(\frac{8+\eta}{12}\right) \delta_{N}^{k-p}+\left(\frac{4-\eta}{24}\right) \delta_{N+1}^{k-p}\right] .
\end{aligned}
$$

The two equations from the boundary conditions are obtained as follows

$$
\begin{aligned}
& \left(\frac{4-\eta}{24}\right) \delta_{-1}^{k+1}+\left(\frac{8+\eta}{12}\right) \delta_{0}^{k+1}+\left(\frac{4-\eta}{24}\right) \delta_{1}^{k+1}=B_{1}\left(t_{k+1}\right), \\
& \left(\frac{4-\eta}{24}\right) \delta_{N-1}^{k+1}+\left(\frac{8+\eta}{12}\right) \delta_{N}^{k+1}+\left(\frac{4-\eta}{24}\right) \delta_{N+1}^{k+1}=B_{2}\left(t_{k+1}\right) .
\end{aligned}
$$

From the equations (3.5)-(3.9), we get the linear system of dimension $(N+3) \times(N+3)$ that can be determined by Mathematica or Matlab. The matrix form can be written as

$$
X \delta^{k+1}=Y\left(b_{k} \delta^{0}+6\left(\delta^{k}\right)^{2}+\sum_{p=0}^{k-1}\left(b_{p}-b_{p+1}\right) \delta^{k-p}\right) .
$$

To start any computation on (3.10), we have the following three equations from the initial condition

$$
\left\{\begin{array}{l}
V_{0}^{\prime}=A_{1}\left(u_{0}\right), \\
V_{j}^{0}=A_{1}\left(u_{j}\right), \quad j=0,1,2, \ldots, N, \\
V_{N}^{\prime}=A_{1}\left(u_{N}\right) .
\end{array}\right.
$$

The above equations can be written in matrix form as

$$
\mathrm{X}_{1} \delta^{0}=\mathrm{Y}_{1}
$$

where

$$
X_{1}=\left[\begin{array}{ccccccc}
-\frac{1}{2 h} & 0 & \frac{1}{2 h} & 0 & \ldots & \ldots & 0 \\
\frac{4-\eta}{24} & \frac{8+\eta}{12} & \frac{4-\eta}{24} & 0 & \ldots & \ldots & 0 \\
0 & \frac{4-\eta}{24} & \frac{8+\eta}{12} & \frac{4-\eta}{24} & \ldots & \ldots & 0 \\
\vdots & \ldots & \ddots & \ddots & \ddots & \ldots & \vdots \\
\vdots & \ldots & \ldots & \ldots & \frac{4-\eta}{24} & \frac{8+\eta}{12} & \frac{4-\eta}{24} \\
0 & \ldots & \ldots & \ldots & -\frac{1}{2 h} & 0 & \frac{1}{2 h}
\end{array}\right]
$$

and $Y_{1}=\left[A_{1}^{\prime}\left(u_{0}\right), A_{1}\left(u_{0}\right), \ldots, A_{1}\left(u_{N}\right), A_{1}^{\prime}\left(u_{N}\right)\right]^{\top}$. All computations are executed in Wolfram Mathematica 12.

\section{Stability}

The stability of suggested technique is carried out using Von Neumann stability analysis. First linearize the non-linear term in equation (3.2) by supposing $v(v-1)=\mu v$, we obtain

$$
v^{k+1}-r v_{u u}^{k+1}+6 \mu r v^{k+1}=b_{k} v^{0}+\sum_{p=0}^{k-1}\left(b_{p}-b_{p+1}\right) v^{k-p} .
$$


Consider the ECuBS difference equation in Fourier series as

$$
\Omega_{j}^{k}=\zeta^{k} e^{i \alpha h j},
$$

where $i=\sqrt{-1}, \alpha, h, \zeta$ are the mode number, the element size and Fourier coefficient, respectively. The equation (4.1) reduces to

$$
\Omega^{k+1}-r \Omega_{\mathfrak{u u}}^{k+1}+6 \mu r \Omega^{k+1}=b_{k} \Omega^{0}+\sum_{p=0}^{k-1}\left(b_{p}-b_{p+1}\right) \Omega^{k-p} .
$$

By expanding the above equation, we get

$$
\begin{aligned}
{[(1+6} & \mu r)\left(\left(\frac{4-\eta}{24}\right) e^{i \alpha(j-1) h}+\left(\frac{8+\eta}{12}\right) e^{i \alpha j h}+\left(\frac{4-\eta}{24}\right) e^{i \alpha(j+1) h}\right)-\frac{r}{24 h^{2}}\left[(2-\eta) e^{i \alpha(j-2) h}\right. \\
+ & \left.\left.4(4+\eta) e^{i \alpha(j-1) h}-6(6+\eta) e^{i \alpha j h}+4(4+\eta) e^{i \alpha(j+1) h}+(2-\eta) e^{i \alpha(j+2) h}\right]\right] \zeta^{k+1} \\
= & b_{k}\left[\left(\frac{4-\eta}{24}\right) e^{i \alpha(j-1) h}+\left(\frac{8+\eta}{12}\right) e^{i \alpha j h}+\left(\frac{4-\eta}{24}\right) e^{i \alpha(j+1) h}\right] \zeta^{0} \\
+ & \sum_{p=0}^{k-1}\left(b_{p}-b_{p+1}\right)\left[\left(\frac{4-\eta}{24}\right) e^{i \alpha(j-1) h}+\left(\frac{8+\eta}{12}\right) e^{i \alpha j h}+\left(\frac{4-\eta}{24}\right) e^{i \alpha(j+1) h}\right] \zeta^{p-k}
\end{aligned}
$$

Dividing (4.2) by $e^{i \alpha h j}$ and combining like terms, we achieve

$$
\begin{aligned}
& {\left[(1+6 \mu r)\left(\frac{8+\eta}{12}+\frac{4-\eta}{12} \cos (\alpha h)\right)-\frac{r}{24 h^{2}}(2(2-\eta) \cos (2 \alpha h)-6(6+\eta)+8(4+\eta) \cos (\alpha h))\right] \zeta^{k+1}} \\
& \times b_{k}\left(\frac{8+\eta}{12}+\frac{4-\eta}{12} \cos (\alpha h)\right) \zeta^{0}+\left(\frac{8+\eta}{12}+\frac{4-\eta}{12} \cos (\alpha h)\right) \sum_{p=0}^{k-1}\left(b_{p}-b_{p+1}\right) \zeta^{k-p} \\
& \Rightarrow \zeta^{k+1}=\frac{1}{w}\left[b_{k} \zeta^{0}+\sum_{p=0}^{k-1}\left(b_{p}-b_{p+1}\right) \zeta^{k-p}\right]
\end{aligned}
$$

$w=1+\frac{r\left[(2-\eta) \sin ^{2}(\alpha h)+4(4+\eta) \sin ^{2}\left(\frac{\alpha h}{2}\right)\right]}{h^{2}(1+6 \mu r)\left(6+(\eta-4) \sin ^{2}\left(\frac{\alpha h}{2}\right)\right)}$. Here $w \geqslant 1$ for $\eta>-2$.

Proposition 4.1. If $\zeta^{\mathrm{k}}, \mathrm{k}=0,1, \ldots, \mathrm{k}$ be the solution of (4.3), then $\left|\zeta^{\mathrm{k}}\right| \leqslant\left|\zeta^{0}\right|$.

Proof. Mathematical induction will be employed. Using $k=0$ in (4.3), we have

$$
\zeta^{1}=\frac{\mathrm{b}_{\mathrm{o}}}{w} \zeta^{0} \leqslant \zeta^{0}, \quad w \geqslant 1, \quad\left|\zeta^{1}\right| \leqslant\left|\zeta^{0}\right| .
$$

Suppose that for $k=0,1, \ldots, M-1,\left|\zeta^{k}\right| \leqslant\left|\zeta^{0}\right|$ is true, then we achieve

$$
\begin{aligned}
\zeta^{k+1} & =\frac{b_{k}}{w} \zeta^{k}+\frac{1}{w} \sum_{p=0}^{k-1}\left(b_{p}-b_{p+1}\right) \zeta^{k-p} \\
\left|\zeta^{k+1}\right| & \leqslant \frac{b_{k}}{w}\left|\zeta^{k}\right|+\frac{1}{w} \sum_{p=0}^{k-1}\left(b_{p}-b_{p+1}\right)\left|\zeta^{k-p}\right| \leqslant \frac{1}{w}\left[b_{k}+\sum_{p=0}^{k-1}\left(b_{p}-b_{p+1}\right)\right]\left|\zeta^{0}\right|=\frac{1}{w}\left|\zeta^{0}\right| \\
\left|\zeta^{k+1}\right| & \leqslant\left|\zeta^{0}\right| .
\end{aligned}
$$

Therefore $\left|\zeta_{j}^{k+1}\right|=\left|\Omega_{j}^{k+1}\right| \leqslant\left|\zeta^{0}\right|=\left|\Omega_{j}^{0}\right|$, so that $\left\|\Omega_{j}^{k+1}\right\|_{2} \leqslant\left\|\zeta^{0}\right\|_{2}$. Hence the proposed method is unconditionally stable. 


\section{Convergence analysis}

Theorem 5.1. Let $v\left(\mathrm{u}, \mathrm{t}^{\mathrm{k}}\right)$ be the exact solution of the given problem (1.1) with initial and boundary conditions (1.2) and let $\mathrm{V}^{\mathrm{k}}$ be the time discrete solution of (4.1), then

$$
\left\|\mathrm{E}^{\mathrm{k}+1}\right\| \leqslant \mathrm{D}+\mathrm{C} \tau^{2-\beta},
$$

where $D$ is a constant and $\mathrm{E}^{\mathrm{k}+1}=v\left(\mathrm{u}, \mathrm{t}^{\mathrm{k}+1}\right)-\mathrm{V}^{\mathrm{k}+1}$.

Proof. Note that the exact solution $v\left(u, t^{k}\right)$ satisfy the equation (4.1), we obtain

$$
v\left(u, t^{k+1}\right)-r v\left(\left(u, t^{k+1}\right)\right)_{u u}+6 \mu r v\left(u, t^{k+1}\right)=b_{k} v\left(u, t^{0}\right)+\sum_{p=0}^{k-1}\left(b_{p}-b_{p+1}\right) v\left(u, t^{k-p}\right)+e_{\tau}^{k+1} .
$$

Subtracting (4.1) from (5.1), we get

$$
E^{k+1}+6 \mu r E^{k+1}-r\left(E^{k+1}\right)_{u u}=b_{k} E^{0}+\sum_{p=0}^{k-1}\left(b_{p}-b_{p+1}\right) E^{k-p}+e_{\tau}^{k+1} .
$$

Since $E^{0}=0$ and taking inner product with $E^{k+1}$ on both sides of the above equation, we obtain

$$
<\mathrm{E}^{\mathrm{k}+1}, \mathrm{E}^{\mathrm{k}+1}>=\mathrm{r}<\mathrm{E}_{\mathfrak{u u}}^{\mathrm{k}+1}, \mathrm{E}^{\mathrm{k}+1}>-6 \mu r<\mathrm{E}^{\mathrm{k}+1}, \mathrm{E}^{\mathrm{k}+1}>+\sum_{\mathrm{p}=0}^{\mathrm{k}-1}\left(\mathrm{~b}_{\mathrm{p}}-\mathrm{b}_{\mathrm{p}+1}\right)<\mathrm{E}^{\mathrm{k}-\mathrm{p}}, \mathrm{E}^{\mathrm{k}+1}>+<\mathrm{e}_{\mathcal{\tau}}^{k+1}, \mathrm{E}^{\mathrm{k}+1}>.
$$

Using the relations $\left\langle v_{\mathfrak{u} u}, v\right\rangle=-\left\langle v_{\mathfrak{u}}, v_{\mathfrak{u}}\right\rangle$ and $\langle v, v\rangle=\|v\|^{2}$, we obtain

$$
\begin{aligned}
& \left\|E^{n+1}\right\|=-r<E_{\mathfrak{u}}^{k+1}, E_{\mathfrak{u}}^{k+1}>-6 \mu r\left\|E^{n+1}\right\|+\sum_{p=0}^{k-1}\left(b_{p}-b_{p+1}\right)<E^{k-p}, E^{k+1}>+<e_{\tau}^{k+1}, E^{k+1}>, \\
& \left\|E^{n+1}\right\|^{2}=-r\left\|E_{\mathfrak{u}}^{k+1}\right\|^{2}-6 \mu r\left\|E^{n+1}\right\|^{2}+\sum_{p=0}^{k-1}\left(b_{p}-b_{p+1}\right)<E^{k-p}, E^{k+1}>+<e_{\tau}^{k+1}, E^{k+1}>.
\end{aligned}
$$

Since $r\left\|E_{\mathfrak{u}}^{k+1}\right\|^{2} \geqslant 0,6 \mu r\left\|E^{n+1}\right\|^{2} \geqslant 0$ and apply Cauchy-Schwarz inequality, $\langle y, z>\leqslant\|y\|\|z\|$, we obtain

$$
\left\|E^{n+1}\right\|^{2} \leqslant \sum_{p=0}^{k-1}\left(b_{p}-b_{p+1}\right)\left\|E^{k-p}\right\|\left\|E^{k+1}\right\|+\left\|e_{\tau}^{k+1}\right\|\left\|E^{k+1}\right\| .
$$

Dividing throughout by $\left\|E^{k+1}\right\|$, we achieve

$$
\begin{aligned}
\left\|E^{n+1}\right\| & \leqslant \sum_{p=0}^{k-1}\left(b_{p}-b_{p+1}\right)\left\|E^{k-p}\right\|+\left\|e_{\tau}^{k+1}\right\| \\
& =D_{1} \sum_{p=0}^{k-1}\left(b_{p}-b_{p+1}\right)+\left\|e_{\tau}^{k+1}\right\|=D_{k}\left(1-b_{k}\right)+\left\|e_{\tau}^{k+1}\right\|<D+C \tau^{2-\beta}, \quad\left(1-b_{k}\right)<1,
\end{aligned}
$$

where $D_{k}=\max _{0 \leqslant p \leqslant k-1}\left\|E^{k-p}\right\|, D=\max _{0 \leqslant k \leqslant M} D_{k}$.

\section{Numerical and graphical results}

Problem 6.1. Consider the following TFFE

$$
{ }_{0}^{C} D_{t}^{\beta} v(u, t)=D_{u u} v(u, t)+6 v(u, t)(1-v(u, t)),
$$

having initial condition

$$
v(u, 0)=\frac{1}{\left(1+e^{\mathfrak{u}}\right)^{2}}
$$


Then, the exact solution of $\beta=1$ is given by

$$
v(u, t)=\frac{1}{\left(1+e^{\mathfrak{u}-5 t}\right)^{2}} .
$$

The (2.2) can be defined as

$$
\begin{aligned}
& V(u, t)=\delta_{j-1} t_{E B} B_{j-1}(u, \eta)+\delta_{j} t E B_{j}(u, \eta)+\delta_{j+1} t E B_{j+1}(u, \eta), \\
& \left(\begin{array}{cc}
0.999999-0.0200429 u+0.0131301 u^{2} & \\
+1.02002 u^{3}-20.5523 u^{4}, & u \in[0.00,0.025) \\
0.999968-0.0163251 u-0.134485 u^{2} & \\
+2.94441 u^{3}-19.6761 u^{4}, & u \in[0.025,0.050) \\
0.999732-0.00227008 u-0.41155 u^{2} &
\end{array}\right. \\
& +4.7108 u^{3}-18.869 u^{4}, \quad u \in[0.050,0.075), \\
& \vdots \quad \vdots \\
& 0.505907+4.50112 u-15.4837 u^{2} \\
& V(u, t)= \begin{cases}+23.6263 u^{3}-13.5007 u^{4}, & u \in[0.425,0.450), \\
0.381458+5.33356 u-17.3459 u^{2} & \\
+025.0332 u^{3}-13.5312 u^{4}, & u \in[0.450,0.475), \\
0.232117+6.28493 u-19.3831 u^{2} & \end{cases} \\
& \begin{array}{l}
0.232117+6.28493 u-19.3831 u^{2} \\
+36.5351 u^{3}-13.6073 u^{4},
\end{array} \quad u \in[0.475,0.500), \\
& \text { : } \\
& -17.4917+78.8991 u-126.27 u^{2} \\
& +89.8092 u^{3}-23.9465 u^{4}, \quad u \in[0.925,0.950), \\
& \begin{array}{l}
-20.5778+89.6769 u-139.789 u^{2} \\
+96.8402 u^{3}-25.1506 u^{4}, \\
-24.1376+101.828 u-154.709 u^{2}
\end{array} \quad u \in[0.950,0.975), \\
& \begin{array}{c}
-24.1376+101.828 u-154.709 u^{2} \\
+104.462 u^{3}-26.4431 u^{4},
\end{array} \quad u \in[0.975,1.00)
\end{aligned}
$$

The above equation (6.1) shows the piecewise solution for $N=40, \tau=100, \beta=0.85$ at $T=3$. Table 1 shows the numerical values for $N=10, \beta=0.3, \beta=0.5, \beta=0.7, \beta=0.9$ at $T=1$. Table 2 displays the computed and exact values for $\tau=200, \beta=1$, at $T=5$. Tables 3 and 4 show that the comparison between exact values and computed values for $\beta=0.99$ at time levels $t=0.4,0.6,0.8,1$. Table 5 demonstrates the approximated solution for different values of $\beta$ and comparison of errors for $\beta=1$ with the results given by Qurashi et al. [12]. Figure 1 depicts the comparison plot for $\beta=1, \tau=100, N=25$ at $T=0.4$. Figures 2 and 3 depict the error plot at different time levels and 3D graph of computed values at $\mathrm{T}=1$.

Table 1: Computed solution for different values of $\beta$ at $T=10$.

\begin{tabular}{lllll}
\hline$u$ & $\beta=0.3$ & $\beta=0.5$ & $\beta=0.7$ & $\beta=0.9$ \\
\hline 0.1 & 0.990625648 & 0.995585274 & 0.998327019 & 0.999665239 \\
0.2 & 0.983695795 & 0.992314926 & 0.997086204 & 0.999416870 \\
0.3 & 0.978870271 & 0.990034574 & 0.996220261 & 0.999243482 \\
0.4 & 0.975937707 & 0.988647671 & 0.995693247 & 0.999137906 \\
0.5 & 0.974798786 & 0.988108861 & 0.995488301 & 0.999096785 \\
0.6 & 0.975458102 & 0.988420797 & 0.995606578 & 0.999120368 \\
0.7 & 0.978023705 & 0.989634135 & 0.996067294 & 0.999212528 \\
0.8 & 0.982714226 & 0.991850701 & 0.996908885 & 0.999380987 \\
0.9 & 0.989874306 & 0.995230050 & 0.998191359 & 0.999637785 \\
\hline
\end{tabular}


Table 2: Comparison of exact solution and computed values for $\beta=1$ at $T=5$.

\begin{tabular}{llll}
\hline$u$ & Exact Sol. & Approximated Sol. & Absolute Error \\
\hline 0.1 & 0.999999999969302 & 0.9999999994371 & $5.32105 \mathrm{E}^{-10}$ \\
0.2 & 0.999999999966074 & 0.9999999990381 & $9.27931 \mathrm{E}^{-10}$ \\
0.3 & 0.999999999962506 & 0.9999999987574 & $1.20507 \mathrm{E}^{-09}$ \\
0.4 & 0.999999999958563 & 0.9999999985840 & $1.37449 \mathrm{E}^{-09}$ \\
0.5 & 0.999999999954206 & 0.9999999985130 & $1.44118 \mathrm{E}^{-09}$ \\
0.6 & 0.999999999949389 & 0.9999999985449 & $1.40447 \mathrm{E}^{-09}$ \\
0.7 & 0.999999999944066 & 0.9999999986861 & $1.25798 \mathrm{E}^{-09}$ \\
0.8 & 0.999999999938184 & 0.9999999989489 & $9.89242 \mathrm{E}^{-10}$ \\
0.9 & 0.999999999931682 & 0.9999999993527 & $5.78989 \mathrm{E}^{-10}$ \\
\hline
\end{tabular}

Table 3: Comparison of exact solution and computed values for $\beta=0.99$ at different time levels.

\begin{tabular}{cllll}
$\mathrm{t}$ & $\mathrm{u}$ & Exact Sol. & Computed Sol. & Absolute Error \\
\hline & 0.1 & 0.756711 & 0.756593 & $1.18501 \mathrm{E}^{-04}$ \\
& 0.3 & 0.714929 & 0.714702 & $2.26861 \mathrm{E}^{-04}$ \\
0.4 & 0.5 & 0.668428 & 0.668201 & $2.27157 \mathrm{E}^{-04}$ \\
& 0.7 & 0.617537 & 0.617366 & $1.70580 \mathrm{E}^{-04}$ \\
& 0.9 & 0.562890 & 0.562818 & $7.22710 \mathrm{E}^{-05}$ \\
\hline & 0.1 & 0.898413 & 0.898174 & $2.38989 \mathrm{E}^{-04}$ \\
& 0.3 & 0.878019 & 0.877455 & $5.64303 \mathrm{E}^{-04}$ \\
0.6 & 0.5 & 0.854038 & 0.853345 & $6.93356 \mathrm{E}^{-04}$ \\
& 0.7 & 0.826057 & 0.825446 & $6.11854 \mathrm{E}^{-04}$ \\
& 0.9 & 0.793708 & 0.793429 & $2.79311 \mathrm{E}^{-04}$ \\
\hline
\end{tabular}

Table 4: Comparison of exact solution and computed values for $\beta=0.99$ at different time levels.

\begin{tabular}{lllll}
\hline $\mathrm{t}$ & $\mathrm{u}$ & Exact Sol. & Computed Sol. & Absolute Error \\
\hline & 0.1 & 0.960713 & 0.960535 & $1.78000 \mathrm{E}^{-04}$ \\
& 0.3 & 0.952328 & 0.951894 & $4.33774 \mathrm{E}^{-04}$ \\
0.8 & 0.5 & 0.942235 & 0.941689 & $5.46185 \mathrm{E}^{-04}$ \\
& 0.7 & 0.930123 & 0.929632 & $4.90582 \mathrm{E}^{-04}$ \\
& 0.9 & 0.915644 & 0.915417 & $2.26741 \mathrm{E}^{-04}$ \\
\hline & 0.1 & 0.985272 & 0.985176 & $1.74580 \mathrm{E}^{-04}$ \\
& 0.3 & 0.982055 & 0.981819 & $2.35580 \mathrm{E}^{-04}$ \\
1.0 & 0.5 & 0.978147 & 0.977848 & $2.98639 \mathrm{E}^{-04}$ \\
& 0.7 & 0.973405 & 0.973136 & $2.69535 \mathrm{E}^{-04}$ \\
& 0.9 & 0.967661 & 0.967536 & $1.25079 \mathrm{E}^{-04}$ \\
\hline
\end{tabular}

Table 5: Comparison between exact solution and computed values for different values of $\beta$ at $(u=0.5)$.

\begin{tabular}{llllll}
\hline$\tau$ & $\beta=0.5$ & $\beta=0.75$ & $\beta=1$ & Error [12] & ECuBS \\
\hline 0.01 & 0.98067674 & 0.99302195 & 0.99999999 & $3.46783 \mathrm{E}^{-08}$ & $1.01028 \mathrm{E}^{-10}$ \\
0.05 & 0.97731227 & 0.99105501 & 0.99999895 & $2.34838 \mathrm{E}^{-05}$ & $8.47740 \mathrm{E}^{-08}$ \\
0.1 & 0.97105617 & 0.98681190 & 0.99982058 & $4.82771 \mathrm{E}^{-04}$ & $2.97365 \mathrm{E}^{-05}$ \\
0.15 & 0.96398140 & 0.98070403 & 0.99758795 & $2.63503 \mathrm{E}^{-03}$ & $5.90773 \mathrm{E}^{-04}$ \\
0.2 & 0.93929810 & 0.95364389 & 0.97260427 & $8.51568 \mathrm{E}^{-03}$ & $5.54255 \mathrm{E}^{-03}$ \\
\hline
\end{tabular}




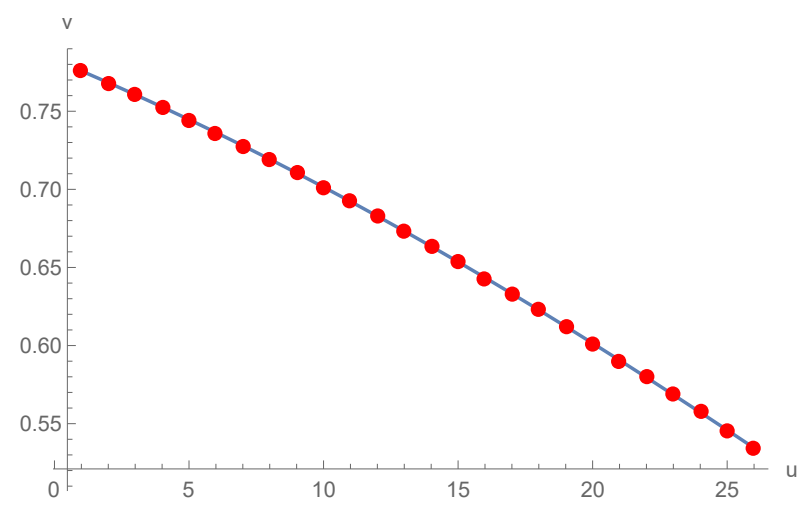

Figure 1: Comparison plot of exact and calculated values at $\mathrm{T}=0.4$.

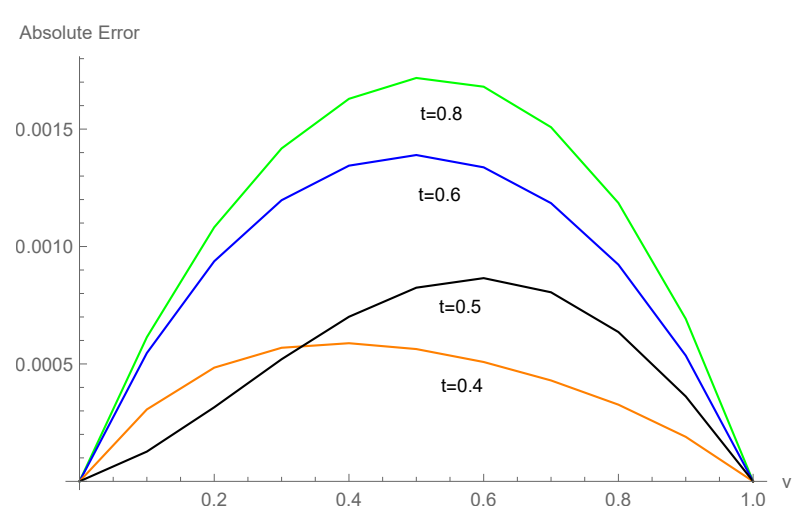

Figure 2: Error plot at different time levels.

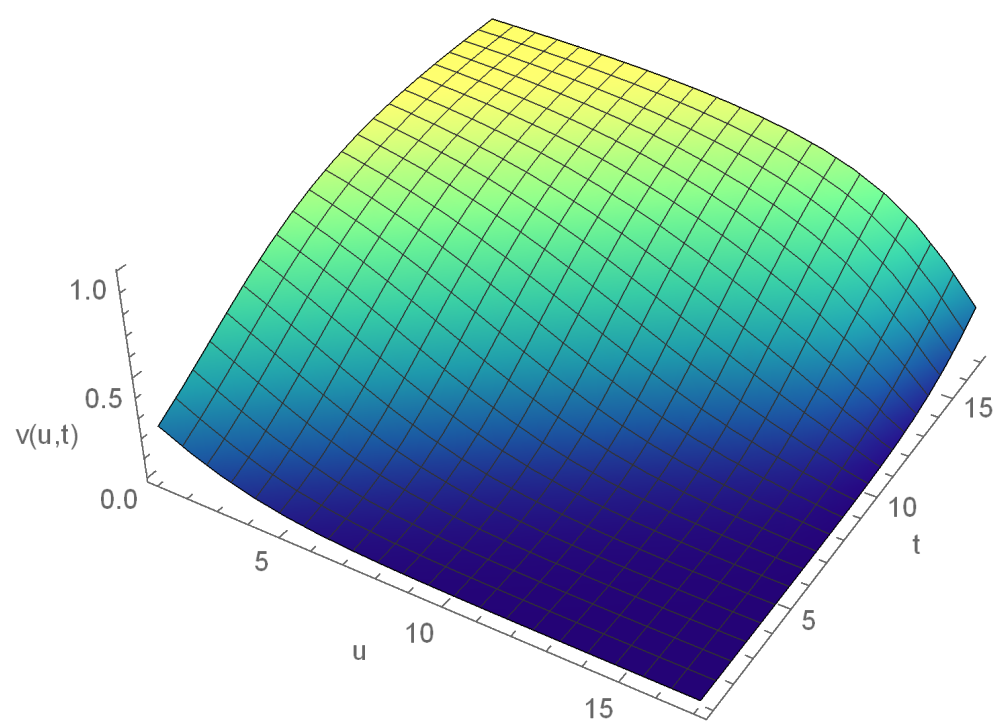

Figure 3: 3D plot of approximated solution at $\mathrm{T}=1$.

\section{Concluding remarks}

The basic objective of this paper is to introduce a numerical algorithm derived from the new ECuBS to find the computational outcomes for non-linear TFFE. The CFD has been used to discretize the fractional derivative in time direction. New ECuBS is utilized in space dimension. The proposed numerical algorithm is convergent and unconditionally stable. From the computational outcomes using the suggested method, we can conclude that these outcomes are in excellent agreement with the exact values and show that this technique can solve the problem efficiently.

\section{Acknowledgment}

The authors are very grateful to the editor and the referees for their helpful comments and valuable suggestions. 


\section{References}

[1] H. F. Ahmed, Efficient methods for the analytical solution of the fractional generalized fisher equation, J. Fract. Calc. Appl., 10 (2019), 85-104. 1

[2] A. Akgül, A novel method for a fractional derivative with non-local and non-singular kernel, Chaos Soliton Fractal, 114 (2018), 478-482. 1

[3] A. Akgül, Reproducing kernel Hilbert space method based on reproducing kernel functions for investigating boundary layer flow of a Powell-Eyring non-Newtonian fluid, J. Taibah Uni. Sci., 13 (2019), 857-863. 1

[4] E. K. Akgül, Solutions of the linear and nonlinear differential equations within the generalized fractional derivatives, Chaos, 29 (2019), 6 pages. 1

[5] A. Akgül, A. Cordero, J. R. Torregrosa, Solutions of fractional gas dynamics equation by a new technique, Math. Meth. Appl. Sci., 43 (2020), 1349-1358. 1

[6] A. Akgül, M. Modanli, Crank-Nicholson difference method and reproducing kernel function for third order fractional differential equations in the sense of Atangana-Baleanu Caputo derivative, Chaos Soliton Fractal, 127 (2019), 10-16. 1

[7] T. Akram, M. Abbas, A. I. Ismail, An extended cubic B-spline collocation scheme for time fractional sub-diffusion equation, AIP Conference Proceedings, 2184 (2019), 15 pages. 1

[8] T. Akram, M. Abbas, A. I. Ismail, Numerical solution of fractional cable equation via extended cubic B-spline, AIP Conference Proceedings, 2138 (2019), 7 pages. 1

[9] T. Akram, M. Abbas, A. I. Ismail, N. H. M. Ali, D. Baleanu, Extended cubic B-splines in the numerical solution of time fractional telegraph equation, Adv. Difference Equ., 2019 (2019), 20 pages. 1

[10] T. Akram, M. Abbas, M. B. Riaz, A. I. Ismail, N. M. Ali, An efficient numerical technique for solving time fractional Burgers equation, Alexand. Eng. J., 2020 (2020), 20 pages. 2.1, 3

[11] T. Akram, M. Abbas, M. B. Riaz, A. I. Ismail, N. M. Ali, Development and analysis of new approximation of extended cubic B-spline to the non-linear time fractional Klein-Gordon equation, Fractals, 2020 (2020), 28 pages. 1

[12] M. M. Al Qurashi, Z. Korpinar, D. Baleanu, M. Inc, A new iterative algorithm on the time fractional Fisher equation: Residual power series method, Adv. Mech. Eng., 9 (2017), 1-8. 1, 6, 5

[13] S. Arshed, Quintic B-spline method for time fractional superdiffusion fourth-order differential equation, Math. Sci., 11 (2017), 17-26. 1

[14] R. L. Bagley, P. J. Torvik, A theoretical basis for the application of fractional calulus to visoelasticity, J. Rheology, 27 (1983), 201-210. 1

[15] D. Baleanu, K. Diethelm, E. Scalas, J. J. Trujillo, Fractional calculus: Models and numerical methods, World Scientific Publishing Co. Pte. Ltd., Hackensack, (2012). 1

[16] D. Baleanu, A. Fernandez, A. Akgül, On a fractional operator combining proportional and classical differintegrals, Mathematics, 8 (2020), 13 pages.

[17] M. Caputo, Elasticita e dissipazione, Zanichelli, Bologna, (1969). 2.1

[18] A. Demir, M. A. Bayrak, E. Ozbilge, An approximate solution of the time fractional fisher equation with small delay by residual power series method, Math. Probl. Eng., 2018 (2018), 8 pages. 1, 1

[19] A. Esen, O. Tasbozan, Numerical solutions of time fractional Burgers equation, Acta Univ. Sapientiae Math., 7 (2015), 167-185. 1

[20] R. A. Fisher, The wave of advance of advantageous genes, Annals of Eugenics, 7 (1937), 355-369. 1

[21] M. M. Khader, K. M. Saad, A numerical study by using the Chebyshev collocation method for a problem of biological invasion: Fractional Fisher equation, Int. J. Biomath, 11 (2018), 15 pages. 1

[22] A. A. Kilbas, H. M. Srivastava, J. J. Trujillo, Theory and applications of fractional differential equations, Elsevier Science B. V., Amsterdam, (2006). 1

[23] R. L. Magin, Fractional calculus in bioengineering, Critical Rev. Biomed. Engineer., 32 (2004), 1-377. 1

[24] R. E. Mickens, Relation between the time and space step-sizes in nonstandard finite difference schemes for the Fishers equation, Numer. Methods Partial Differential Equations, 13 (1997), 51-55. 1

[25] S. T. Mohyud-Din, T. Akram, M. Abbas, A. I. Ismail, N. H. M. Ali, A fully implicit finite difference scheme based on extended cubic B-splines for time fractional advection-diffusion equation, Adv. Difference Equ., 2018 (2018), 17 pages. 1

[26] Z. Odibat, Approximations of fractional integrals and Caputo fractional derivatives, Appl. Math. Comput., 178 (2006), 527-533. 1

[27] D. Olmos, B. D. Shizgal, A pseudo-spectral method of solution of Fisher's equation, J. Comput. Appl. Math., 193 (2006), 219-242. 1

[28] D. A. Robinson, The use of control systems analysis in the neurophysiology of eye movements, Annual Rev. Neurosci., 4 (1981), 463-501. 1

[29] S. G. Samko, A. A. Kilbas, O. I. Marichev, Fractional Integrals and Derivatives: Theory and Applications, Gordon and Breach, New York, (1993). 1

[30] O. Tasbozan, A. Esen, Quadratic B-spline Galerkin method for numerical solutions of fractional telegraph equations, Bulletin Math. Sci. Appl., 18 (2017), 23-39. 1

[31] R. Uddin, Comparison of the nodal integral method and non standard finite-difference schemes for the Fisher equation, SIAM J. Sci. Comput., 22 (2001), 1926-1942. 1 
[32] P. Veeresha, D. G. Prakasha, H. M. Baskonus, Novel simulations to the time-fractional Fisher's equation, Math. Sci., 13 (2019), 33-42. 1

[33] X. Zhang, J. Liu, An analytic study on time fractional Fisher equation using homotopy perturbation method, Walailak J. Sci. Tech., 11 (2014), 975-985. 1, 1 